\title{
Capacity Performance of Overhead Transmission Multiple-Input Multiple-Output Broadband over Power Lines Networks: The Insidious Effect of Noise and the Role of Noise Models
}

\author{
Athanasios G. Lazaropoulos* \\ School of Electrical and Computer Engineering / National Technical University of Athens / 9 Iroon \\ Polytechniou Street / Zografou, GR 15780
}

Received May 7, 2016; Accepted May 30, 2016; Published June 6, 2016

\begin{abstract}
Extending the analysis already presented in [1], this paper considers broadband potential of overhead (OV) transmission multiple-input multiple-output (MIMO) broadband over power lines (BPL) networks when different noise conditions occur and different well-proven noise models are adopted.

The contribution of this paper is two-fold. First, the broadband potential of a great number of indicative OV high-voltage (HV) BPL topologies and of MIMO transmission schemes is studied in terms of appropriate capacity metrics. The relevant numerical results reveal the significant dependence of MIMO capacity metrics on noise conditions. Second, various well-known BPL noise models from the literature are compared on the basis of their achieved OV HV MIMO BPL capacity. Through the careful study of the capacity results of noise models, it is demonstrated that spectrally flat additive white Gaussian noise (AWGN) may be comfortably assumed as an efficient noise model in transmission MIMO BPL networks. Also in MIMO BPL networks, the comparative capacity analysis of noise models shows small differences among them in the $3-88 \mathrm{MHz}$ frequency range.
\end{abstract}

Keywords: Smart Grid; Broadband over Power Lines (BPL) Networks; Power Line Communications (PLC); Noise Models; Transmission Power Grids; Capacity; Multiple-Input Multiple-Output (MIMO) Systems

\section{Introduction}

The deployment of broadband over power lines (BPL) networks across the overhead high-voltage (OV HV) power grid is among the first steps towards its modernization. Some of the futuristic characteristics of the new transmission power grid are: (i) its IP-based communications network capabilities; (ii) a great number of smart grid applications; and (iii) the potential of broadband last mile access through its wired/wireless interfaces [1]-[13].

However, recent studies outline that today's OV HV BPL networks are not providing sufficient bandwidth for supporting the latest ultra-broadband applications (such as high-definition television, voice over internet protocol, and console gaming) or some state-of-art sophisticated smart grid applications (such as energy trade, electric vehicle power management, etc) in a reliable way [7], [9], [11], [14], [15]. Towards that direction, the integration of today's OV HV BPL networks -i.e., single-input single- 
output (SISO) ones- with multiple-input multiple-output (MIMO) technology features, which are firstly presented in [12], [13], [16]-[21], is decisive for the further deployment of OV HV BPL networks.

When considered as a transmission medium for communications signals, OV HV BPL networks -either SISO or MIMO ones- are subjected to various inherent deficiencies such as high and frequency-selective attenuation and noise [3]-[7]. Each of the aforementioned adverse factors critically influences the broadband potential of OV HV BPL networks [19], [22]. Due to the upgrade of the existing SISO BPL networks to MIMO ones across the entire power grid, the development of accurate and efficient models that are suitable for describing either the channel attenuation or the noise of MIMO BPL networks at high frequencies is imperative.

As concerns the channel attenuation, the well-established hybrid method, which is reliably employed to examine the behavior of various multiconductor transmission line (MTL) structures, is also adopted in this paper [2]-[13], [23]-[25]. Here, it should be noted that the hybrid method has been already successfully tested in transmission and distribution MIMO BPL networks [13], [26]. In fact, this hybrid method is based on: (i) a bottom-up approach that consists of the MTL theory and various similarity transformations, such as eigenvalue (EVD) and singular value (SVD) decompositions [3], [10], [11], [19], [23], [25], [27], [28]; and (ii) a top-down approach, which is denoted as TM2 method, that is determined through the concatenation of multidimensional chain scattering matrices of the MIMO BPL network modules [2]-[11], [24], [27].

Except for the channel attenuation and its relevant hybrid method, to determine the capacity of OV HV MIMO BPL networks, there is the need of an appropriate model to describe the noise that occurs across OV HV networks [2]-[8], [29]. In fact, many researchers have focused their efforts on deriving suitable noise models [22], [30]-[36]. Despite the significant efforts towards modeling of BPL noise in the $3-88 \mathrm{MHz}$ frequency range, a simple, but yet accurate and universal model is not still widely acceptable [37]-[40]. Anyway, as capacity computations are concerned, many BPL researchers tend to use simple but effective noise models that are proven to have realistic, validated with measurements and comparable results in the $3-88 \mathrm{MHz}$ frequency range [1], [30], [41]-[43]. Another common practical procedure among BPL engineers is the consideration of spectrally flat additive white Gaussian noise (AWGN) -denoted as FL noise model in this paper- [4], [6], [12], [23], [29], [44]. Apart from the distribution SISO BPL networks that have been examined in [1], the validity and the accuracy of the FL noise model are now investigated in OV HV MIMO BPL networks with reference to the other well-verified noise models of the literature.

The rest of this paper is organized as follows: In Sec. II, the OV transmission MTL configuration and the respective indicative topologies are presented. Sec. III summarizes the principles of MIMO BPL transmission through the lens of the well-validated hybrid method. Sec. IV recapitulates the main factors that influence MIMO BPL capacity such as electromagnetic interference (EMI) policies and BPL noise characteristics. Special mention is given to the BPL noise models that are available from [1]. In Sec. V, numerical results and discussion validate the effect of noise as well as the significance of accurate BPL noise models when different OV HV BPL topologies and MIMO transmission schemes occur. Sec. VI concludes this paper. 


\section{Transmission Power Grids OV HV MTL Configuration}

OV $400 \mathrm{kV}$ double-circuit transmission phase lines with radii $r_{\mathrm{p}}^{\mathrm{OVHV}}=15.3 \mathrm{~mm}$ hang at typical heights $h_{\mathrm{p}}^{\text {OVHV }}$ equal to $20 \mathrm{~m}$ above ground -i.e., conductors $1,2,3,4,5$, and 6-. These six phase conductors are divided into three bundles; the phase conductors of each bundle are connected by non-conducting spacers and are separated by $\Delta_{\mathrm{p} 2}^{\mathrm{OVHV}}$ equal to $400 \mathrm{~mm}$, whereas bundles are spaced by $\Delta_{\mathrm{p} 1}^{\mathrm{OVHV}}$ equal to $10 \mathrm{~m}$. Except for the phase conductors, two parallel neutral conductors, which are the conductors 7 and 8 , hang at heights $h_{\mathrm{n}}^{\text {OVHV }}$ equal to $23.7 \mathrm{~m}$. The neutral conductors with radii $r_{\mathrm{n}}^{\text {OVHV }}=9 \mathrm{~mm}$ are spaced by $\Delta_{\mathrm{n}}^{\mathrm{OVHV}}$ equal to $12 \mathrm{~m}$. Schematically, the OV HV double-circuit MTL configuration of the aforementioned eight conductors $\left(n^{\mathrm{OVHV}}=8\right)$, which is considered in the present work, is given in Fig. 1.

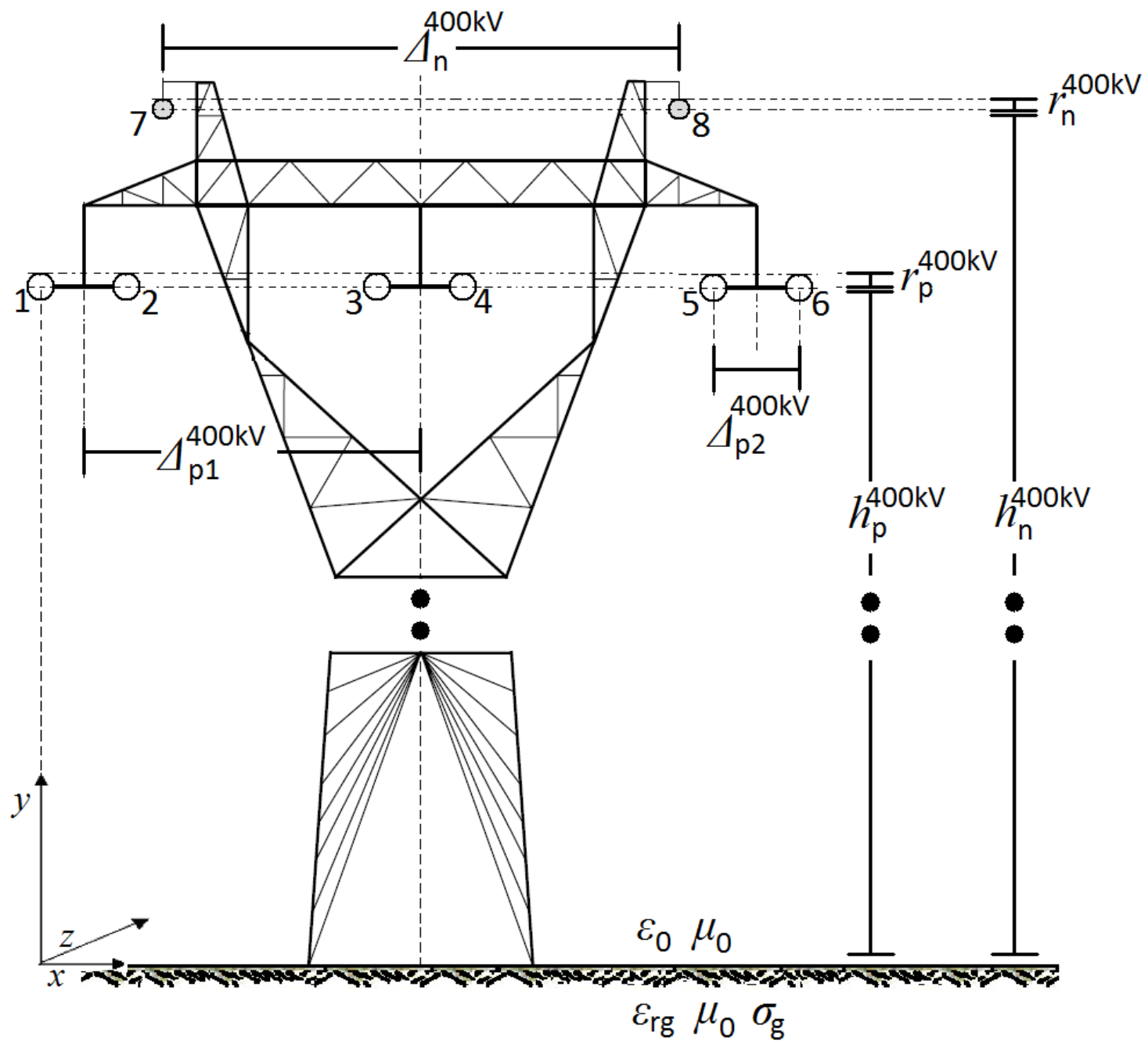

Figure 1. OV 400kV double-circuit MTL configuration [9]. 
In accordance with [7], [9], [44], [45], the ground with conductivity $\sigma_{\mathrm{g}}=5 \mathrm{mS} / \mathrm{m}$ and relative permittivity $\varepsilon_{\mathrm{rg}}=13$ is considered as the reference conductor. The aforementioned ground parameters define a realistic scenario during the following analysis while the impact of imperfect ground on broadband signal propagation via $\mathrm{OV}$ HV power lines was analyzed in [3]-[9], [23], [44], [46], [47].

\section{Indicative OV HV BPL Topologies}

The simple BPL topology of Fig. 2, being bounded by the transmitting and receiving ends and having $N$ branches, is considered. To apply the hybrid method, the simple BPL topology of Fig. 2 is separated into segments (network modules), each of them comprising the successive branches encountered [10], [11].

In accordance with [2]-[13], [23], [24], [27], [30], [44], [48] and with reference to Fig. 2, average path lengths of the order of $25 \mathrm{~km}$ are considered in OV HV BPL topologies. The following four indicative OV HV BPL topologies concerning end-to-end connections of average path lengths that are detailed in [7], [9] are examined, namely:

1. A typical urban topology (OV HV urban case);

2. A typical suburban topology (OV HV suburban case);

3. A typical rural topology (OV HV rural case); and

4. The "LOS" transmission along the same end-to-end distance $L=L_{1}+\ldots+L_{N+1}=25 \mathrm{~km}$. This topology corresponds to Line of Sight transmission in wireless channels.

Besides the characteristics of OV HV BPL topologies, some circuital parameters need to be highlighted [2]-[13], [23], [24], [27], [30], [44], [45], [48]. More specifically, the branching cables are assumed identical to the transmission cables and the interconnections between the transmission and branch conductors are fully activated. The transmitting and the receiving ends are assumed matched to the characteristic impedance of the modal channels, whereas the branch terminations are assumed open circuit.

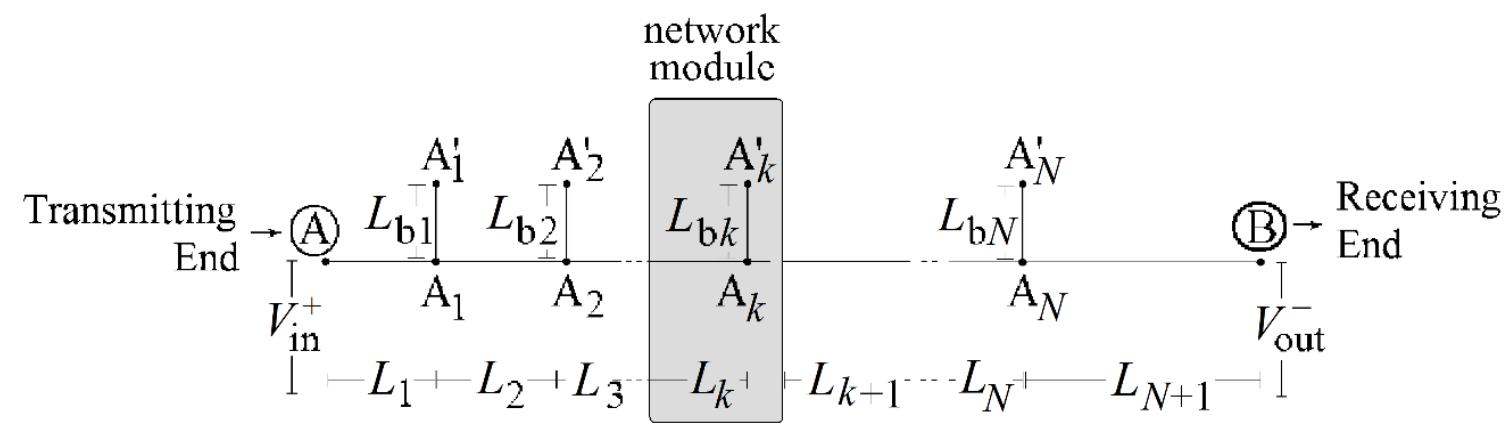

Figure 2. End-to-end HV/BPL connection with $\mathrm{N}$ branches and a network module [9].

\section{The Basics of BPL Transmission Analysis MTL Theory and EVD Modal Analysis - A Briefing}

As it has already been mentioned in [1]-[11], [23], [44], through a matrix approach, the standard TL analysis can be extended to the MTL case. Compared to a twoconductor line supporting one forward- and one backward-traveling wave, an MTL 
structure with $n^{\mathrm{OVHV}}+1$ conductors parallel to the $\mathrm{z}$ axis as depicted in Fig. 1 may support $n^{\mathrm{OVHV}}$ pairs of forward- and backward-traveling waves with corresponding propagation constants. Each pair of forward- and backward-traveling waves is referred to as a mode [2]-[11], [23], [28], [44].

The spectral behavior of the $n^{\text {OVHV }}$ modes, which are supported by the aforementioned MTL configurations and propagate across OV HV BPL topologies, is thoroughly investigated in [1]-[11], [23]-[25], [27], [28], [44]-[49]. Through TM2 method, which is based on the scattering matrix theory and presented analytically in [10], [11], their spectral behavior is modeled proposing the $n^{\text {OVHV }} \times n^{\text {OVHV }}$ EVD modal transfer function matrix $\left.\mathbf{H}^{\mathrm{m}}\{\}\right\}$ whose elements $H_{i, j}^{\mathrm{m}}\left\{\{\}, i, j=1, \ldots, n^{\mathrm{OVHV}}\right.$ are the EVD modal transfer functions where $H_{i, j}^{\mathrm{m}}$ denotes the element of matrix $\mathbf{H}^{\mathrm{m}}\{\cdot\}$ in row $i$ of column $j$.

Through appropriate similarity transformations, which are presented in [1]-[11], [23], [44], the $n^{\text {OVHV }} \times n^{\text {OVHV }}$ channel transfer function matrix $\mathbf{H}\{-\}$ that relates line quantities with EVD modal ones is determined from

$$
\mathbf{H}\{\cdot\}=\mathbf{T}_{V} \cdot \mathbf{H}^{m}\{\cdot\} \cdot \mathbf{T}_{V}^{-1}
$$

where $\mathbf{T}_{V}$ is $n^{\text {OVHV }} \times n^{\text {OVHV }}$ matrix depending on the frequency, the power grid type, the physical properties of the cables and the geometry of the MTL configuration.

\section{MIMO BPL Networks and SVD Modal Analysis}

Based on the multiple application of $\mathrm{WtG}$ coupling schemes at transmitting and receiving ends of OV HV BPL topologies, which is analytically described in [1], [12], [13], the number of active transmit ports $n_{T}$ and receive ports $n_{R}$ may vary from one to $n^{\text {OVHV }}$. Appropriately decomposing channel transfer function matrix $\mathbf{H}\{\cdot\}$ of eq. (1) by using SVD transformations, $\min \left\{n_{T}, n_{R}\right\}$ parallel and independent SISO BPL channels may be defined, namely [12], [13], [16], [17], [20], [21], [50], [51]:

$$
\widetilde{\mathbf{H}}^{m}\{\cdot\}=\widetilde{\mathbf{T}}_{V}^{\mathrm{H}} \cdot \mathbf{H}^{+}\{\cdot\} \cdot \widetilde{\mathbf{T}}_{I}
$$

where

$$
H_{i j}^{+}\{\cdot\}=\left\{\begin{array}{cc}
H_{i j}\{\cdot\} & \text { if }\left(i \in N_{T} \text { and } j \in N_{R}\right) \\
0 & \text { else }
\end{array} i, j=1, \ldots, n^{\text {OVHV }}\right.
$$

denotes the element of matrix $\mathbf{H}^{+}\{\cdot\}$ in row $i$ of column $j$. From eqs. (2) and (3), $\mathbf{H}^{+}\{\cdot\}$ is the $n^{\text {OVHV }} \times n^{\text {OVHV }}$ extended channel transfer function matrix whose elements $H_{i j}^{+}\left\{\{\}, i, j=1, \ldots, n^{\mathrm{OVHV}}\right.$ are the extended channel transfer functions of OV HV MIMO BPL topologies, $N_{T}$ and $N_{R}$ are the active transmit port and the active receive port sets, respectively, $\quad \widetilde{\mathbf{H}}^{m}\{\}$ is a diagonal matrix operator whose elements $\widetilde{H}_{i}^{m}\{\cdot\}, i=1, \ldots, \min \left\{n_{T}, n_{R}\right\}$ are the singular values of $\mathbf{H}^{+}\{\cdot\}$ and, at the same time, the SVD modal transfer functions, $\min \{x, y\}$ returns the smallest value between either $\mathrm{x}$ or $\mathrm{y}$, []$^{\mathrm{H}}$ denotes the Hermitian conjugate of a matrix, and $\widetilde{\mathbf{T}}_{V}$ and $\widetilde{\mathbf{T}}_{I}$ are $n^{\mathrm{OVHV}} \times n^{\mathrm{OVHV}}$ unitary matrices [12], [13], [16], [20]. 


\section{EMI Policies, Noise and Capacity of Transmission MIMO BPL Networks EMI Policies}

As it has already been analyzed in [1], a great number of regulatory bodies has established proposals (EMI policies) concerning the electromagnetically safe BPL operation. Among these, FCC Part 15 is the most noted one and is analytically presented in [32], [33], [52]-[54]. The injected power spectral density limits (IPSD limits) proposed by Ofcom for compliance with FCC Part 15 are adopted in this paper [32], [33]. More specifically, in the $3-30 \mathrm{MHz}$ and $30-88 \mathrm{MHz}$ frequency ranges, respective maximum levels of $-60 \mathrm{dBm} / \mathrm{Hz}$ and $-77 \mathrm{dBm} / \mathrm{Hz}$ constitute appropriate IPSD limit $p(f)$ for OV HV BPL connections providing presumption of compliance with the current FCC Part 15 [1]-[13], [32], [33], [53], [54]. Without losing the generality of the analysis, a typical procedure is the consideration of common IPSD limits for OV transmission and distribution BPL networks (either SISO or MIMO) exploiting the significant similarities regarding OV BPL transmission.

\section{Noise Characteristics}

Regardless of the power grid type -either SISO or MIMO, either OV or underground (UN), either HV or medium-voltage (MV) or low-voltage (LV)-, BPL noise may be considered as the superposition of five noise types, namely [1]-[11], [22], [30]-[36], [39], [41]-[43], [52], [55]-[58]: (i) the colored background noise; (ii) the narrowband noise; (iii) the periodic impulsive noise asynchronous to the mains frequency; (iv) the periodic impulsive noise synchronous to the mains frequency; and (v) the asynchronous impulsive noise. As it has already been reported in [1] and analyzed in [4], [6], [12], [13], [23], [29], [44], only the non resolvable limitations should be considered during the BPL capacity computations. Since only the first two types of noise can be treated as background noise due to their quasi-stationary behavior either in frequency- or in time-domain, the last three types of noise, which are treated as impulsive noise, are not taken into account during the following capacity computations.

As it has already presented in [1], to describe this noise PSD, a first-order exponential function is used and is determined from [41], [56], [57]

$$
N(f)=N_{\mathrm{NF}}+n_{1} e^{-n_{2} f}
$$

where $f$ is the frequency in $\mathrm{MHz}, N_{\mathrm{NF}}$ is the spectrally flat AWGN/PSD expressed in $\mathrm{dBm} / \mathrm{Hz}$, and $n_{1}$ and $n_{2}$ are the exponential coefficients of the noise model.

As it regards the AWGN properties of transmission BPL networks in $3-88 \mathrm{MHz}$ frequency range, the AWGN/PSD level $N_{\mathrm{NF}}$ is assumed equal to $-105 \mathrm{dBm} / \mathrm{Hz}$ [2]-[13], [22], [23], [29], [30], [32]-[36], [44], [56]. Apart from this value that represents the average noise type, AWGN/PSD levels may vary from $-120 \mathrm{~dB}$ (noise type A) to $-90 \mathrm{~dB}$ (noise type B) representing the good and the bad noise scenario. This noise PSD variance of $15 \mathrm{~dB}$ from average conditions gives a representative image of the variety of noise environments that may occur in transmission BPL networks [2]-[13], [29]. Similarly to IPSD limits, common noise characteristics are assumed for OV HV, OV MV and OV LV BPL networks which is a rather common procedure.

To examine the noise impact on OV HV MIMO BPL capacity, the seven different noise models of [1] are also examined in this paper: 
1. the FL noise model that is the spectrally flat AWGN model where $n_{1}$ and $n_{2}$ are equal to zero (proposed by [1], [4], [6], [12], [23], [29], [44]);

2. the OPERA noise model that is suitable for outdoor urban and suburban noise environments (presented in [30]);

3. the MEN noise model (presented in [42], [43]);

4. the PH1 noise model that is appropriate for urban noise conditions (presented in [41], [56], [57]);

5. the PH2 noise model that is focused on industrial noise environments (presented in [41], [56], [57]);

6. the ESM1 noise model (presented in [58]); and

7. the ESM2 noise model that is appropriate for urban noise conditions (presented in [58]).

In Fig. 3, the noise PSD of the seven aforementioned noise models is plotted versus frequency in the $3-88 \mathrm{MHz}$ frequency range. Observations of background noise in the $3-88 \mathrm{MHz}$ frequency range reveal that its noise PSD is higher at lower frequencies. Actually, the noise PSDs of the noise models present significant $\mathrm{dB}$ differences at lower frequencies. Conversely, as frequency increases, noise PSD of background noise reduces and tends to spectrally flat AWGN. Indeed, the noise PSDs of the noise models tends to coincide at higher frequencies [22], [34]-[36], [39], [41], [42], [55]-[58].

\section{Capacity}

Capacity is defined as the maximum achievable transmission rate that can be reliably transmitted over a BPL network and depends on the applied MTL configuration, the type of power grid, the power grid topology, the adopted EMI policy, the noise environment and the adopted MIMO transmission scheme [2]-[13]. 


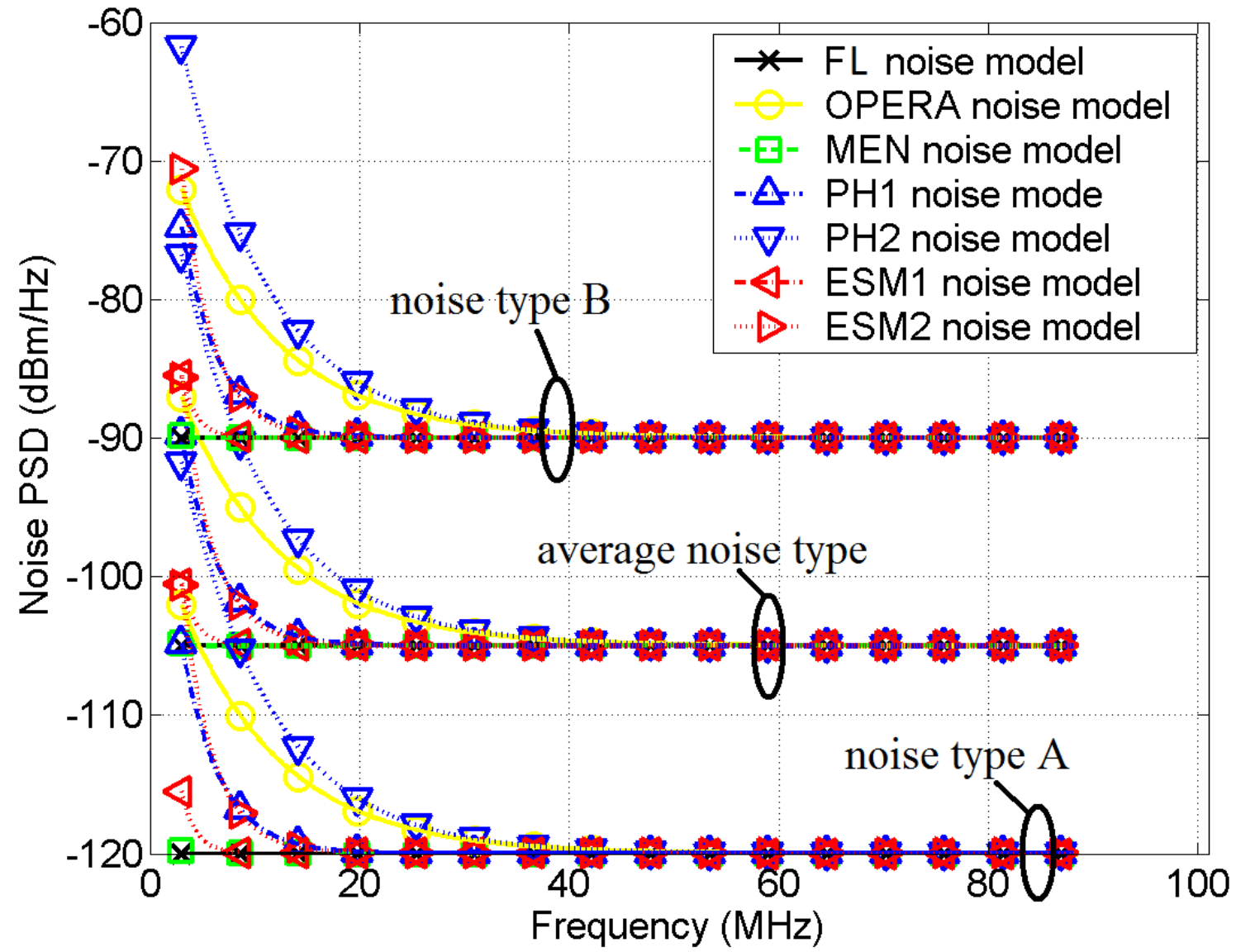

Figure 3. Noise PSD of the indicative noise models in the $3-88 \mathrm{MHz}$ frequency band when noise type $A$, average noise type and noise type $B$ are assumed.

Based on eqs. (1)-(3) and with reference to Fig. 2, the overall capacity $C$ of OV HV MIMO BPL networks is determined from [4], [6], [12], [13], [16]-[21], [23]

$$
C_{\mathrm{A} \rightarrow \mathrm{B}}^{\text {MIMO }}=f_{s} \sum_{q=0}^{Q-1} \sum_{i=1}^{\min \left\{n_{R}, n_{T}\right\}} \log _{2}\left\{1+\frac{\left\langle p\left(q f_{s}\right)\right\rangle_{L}}{n_{T} \cdot\left\langle N\left(q f_{s}\right)\right\rangle_{L}} \cdot\left|\widetilde{H}_{i}^{m}\left(q f_{s}\right)\right|^{2}\right\}
$$

where $[\cdot]_{\mathrm{A} \rightarrow \mathrm{B}}$ determines the transmitting (A) and receiving (B) end, $\langle\cdot\rangle_{L}$ is an operator that converts $\mathrm{dBm} / \mathrm{Hz}$ into a linear power ratio $(\mathrm{W} / \mathrm{Hz}), Q$ is the number of subchannels in the BPL signal frequency range of interest, and $f_{s}$ is the flat-fading subchannel frequency spacing. Note that eq. (5) is based on the use of equal power uncorrelated sources as the common case is assumed; say, the transmitting end does not have channel state information.

Based on eq. (5), the spectral efficiency metric of cumulative capacity is applied in order to assess the broadband potential of OV HV MIMO BPL connections [1], [4], [6]. The cumulative capacity is defined as the cumulative upper limit of information which can be reliably transmitted over a BPL network. 


\section{Numerical Results and Discussion}

The numerical results of various types of OV HV MIMO BPL networks aim at investigating: (a) their broadband potential through the integration of MIMO features; (b) the influence of different noise environments on the capacity performance; and (c) the role of noise models as well as the accuracy degree of simpler ones.

As the following capacity analysis is concerned, only full MIMO transmission schemes -i.e., SISO, 2x2 MIMO, 3x3 MIMO, 4x4 MIMO, 5x5 MIMO, 6x6 MIMO, $7 \times 7$ MIMO and $8 \times 8$ MIMO ones- will be examined in the rest of this paper due to their favorable capacity characteristics in comparison with corresponding single-input multiple-output (SIMO) and multiple-input single-output (MISO) transmission scheme alternatives. Note that, the full MIMO degree, which is the number of freedom degrees of an applied full MIMO transmission scheme, is equal to 1, 2, 3, 4, 5, 6, 7 and 8 for the eight considered full MIMO transmission schemes, respectively. Finally, among the possible configurations for a given full MIMO transmission scheme, only the one with the maximum capacity performance is examined (for more details see in [12], [13], [26]). These considerations do not affect the generality of the following OV HV MIMO BPL capacity analysis, anyway [2], [7]-[12].

\section{How MIMO Transmission Schemes Blow Up the Capacity Balloon whereas Disturbed Noise Environments Deflate it}

Today's OV HV SISO BPL networks are not providing adequate throughput so as to cover future bandwidth-demanding smart grid applications in a trustworthy way [7], [9], [11]. Thus, the deployment of MIMO transmission schemes across OV HV BPL networks is an one-way procedure [12], [13], [16]-[21]. Nevertheless, noise critically degrades BPL systems performance. Due to its highly variable nature, the detailed knowledge of the noise properties in the future's mainstream BPL operation bands, such as the $3-88 \mathrm{MHz}$ frequency range, may contribute towards the design of more efficient modulation and coding schemes suitable for OV HV MIMO BPL networks.

Therefore, the following subsection of this paper focuses on the capacity capabilities in terms of cumulative capacity in the $3-88 \mathrm{MHz}$ frequency band when these two contradictious factors -i.e., MIMO technology and noise- are deployed in accordance with FCC Part 15 limits. In order to evaluate the effect of different noise conditions on OV HV MIMO BPL capacity, apart from the average noise type, noise type $A$ and B, which have been presented in Sec. IVB, are assumed. Finally, in this subsection, only the FL noise model is applied.

In Fig. 4(a), the cumulative capacity is plotted versus frequency for the indicative OV HV BPL topologies, presented in Sec. IIB, when SISO transmission scheme is applied. In Figs. 4(b)-(h), similar curves are given in the case of 2x2 MIMO, 3x3 MIMO, 4x4 MIMO, 5x5 MIMO, 6x6 MIMO, 7x7 MIMO and 8x8 MIMO transmission schemes, respectively.

Figs. 4(a)-(h) validate the incredible capacity boost of OV HV BPL networks by appropriately exploiting the eight wires of their OV MTL configurations. It is evident that MIMO technology introduces a new abundant capacity era for BPL systems and, at the 

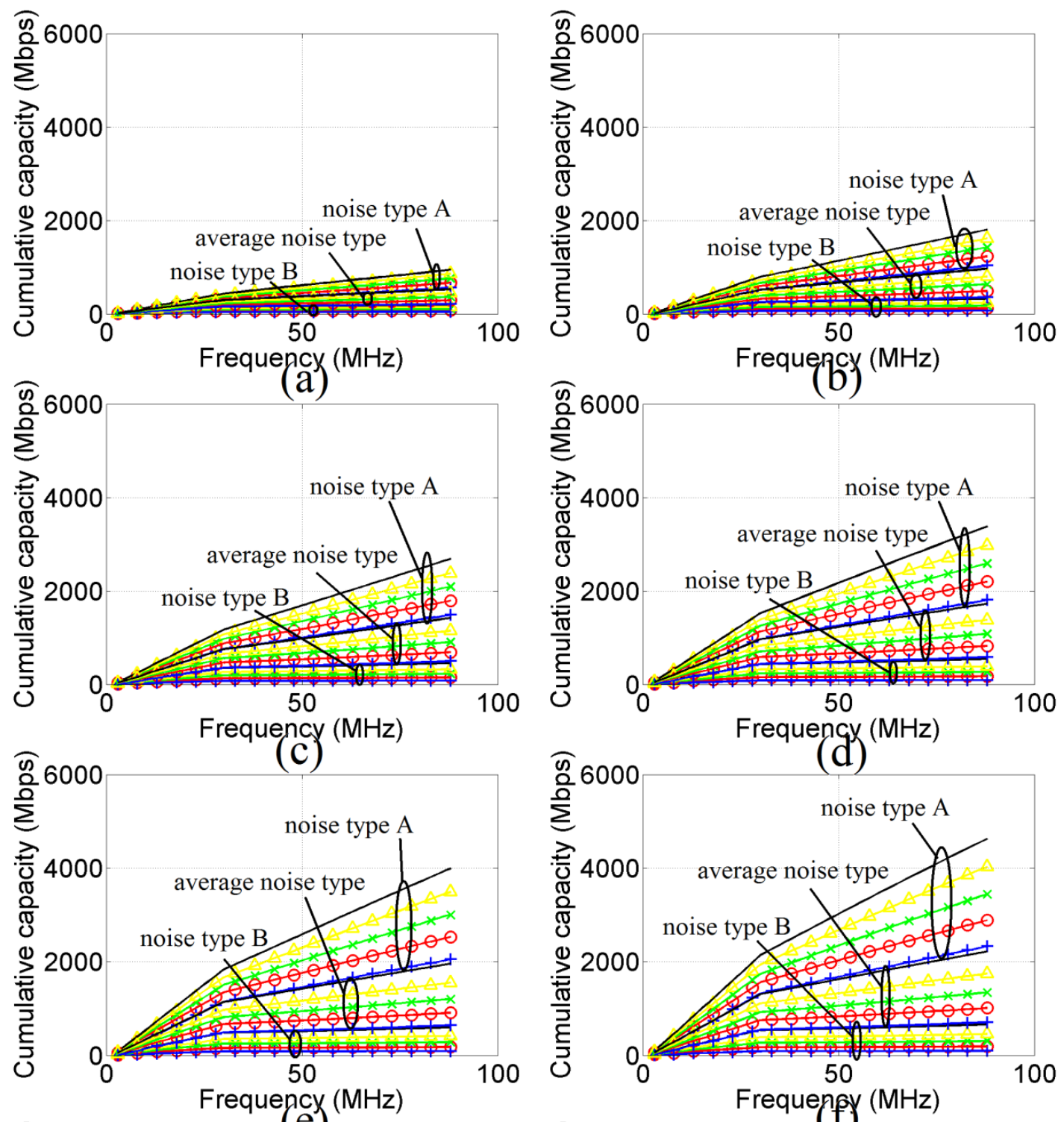

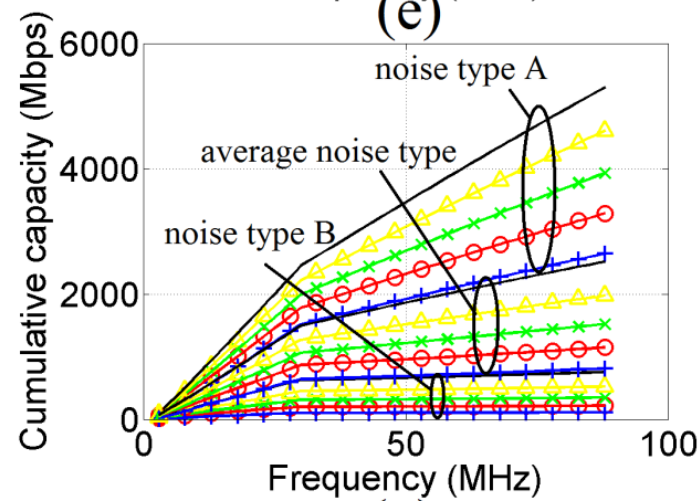

(g)

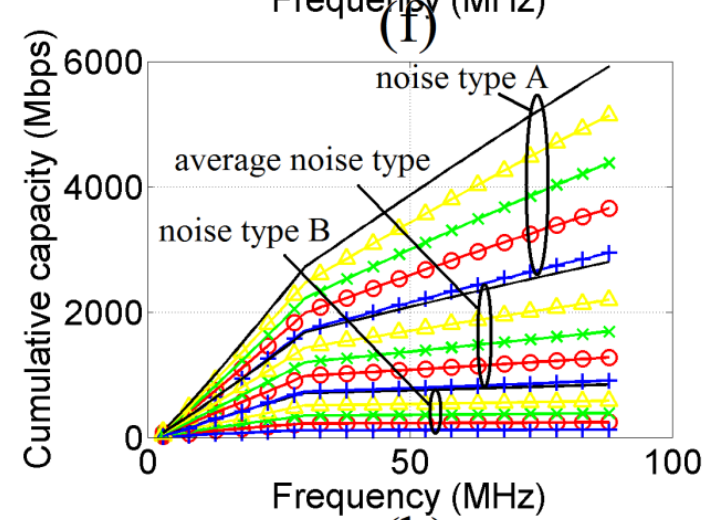

(h)

Figure 4. Cumulative capacity versus frequency of the four indicative topologies of OV HV BPL networks -urban case $A(\theta)$, suburban case $(*)$, rural case $(\triangle)$, and "LOS" case $(-)$ in the $3-88 \mathrm{MHz}$ frequency band for different MIMO transmission schemes when FCC Part 15 is adopted. Average noise type, noise type A, and noise type $B$ are considered. (a) SISO. (b) $2 \times 2$ MIMO. (c) 3x3 MIMO. (d) 4x4 MIMO. (e) 5x5 MIMO. (f) 6x6 MIMO. (g) 7x7 MIMO. (h) 8x8 MIMO. 
same time, transforms OV HV BPL networks into the leading broadband technology among the other candidate technologies in the oncoming smart grid. Actually, when favorable noise conditions occur, OV HV MIMO BPL networks are upgraded to multi-Gbps networks reaching a 6Gbps capacity ceiling; this broadband potential sets BPL technology as either fiber-optic alternative or backhaul network for other surrounding wired/wireless technologies [59], [60]. Anyway, during the comparison of OV HV BPL technology with other competitive broadband technologies, it is worth pointing out that OV HV BPL networks are a potentially excellent communications medium offering these high capacity characteristics over a $25 \mathrm{~km}$ repeater span well beyond $88 \mathrm{MHz}$ having the advantage of no additional wiring.

Concurrently, Figs. 4(a)-(h) unveil the insidious role of noise in today's and future's transmission BPL networks [22], [34], [56]. In all the cases examined, capacity differences of the order of hundreds of Mbps -even of Gbps in some cases- are observed when disturbed noise environments occur. Actually, a 15dB-increase of noise PSD (noise type B) in the $3-88 \mathrm{MHz}$ frequency band corresponds to the recall of OV HV 8x8 MIMO BPL network capacity results to the levels of OV HV SISO transmission schemes; an average capacity loss of the order of $1.3 \mathrm{Gbps}$. In [1], it has been reported that an appropriate rule of thumb for distribution SISO BPL networks suggests that each $\mathrm{dB}$ of increase above average noise PSD corresponds to an average capacity reduction that is approximately equal to $23 \mathrm{Mbps}$ in the $3-88 \mathrm{MHz}$ frequency range. Similarly to [1] and by observing Figs. 4(a)-(h), this rule of thumb may be extended in order to cope with OV HV MIMO BPL networks in the same frequency range; each $\mathrm{dB}$ of increase above average noise PSD per each full MIMO degree corresponds to an average capacity reduction that is approximately equal to $13 \mathrm{Mbps}$, which is significantly lower compared to the distribution SISO BPL case. Similar deductions may be drawn regarding capacity increase through the decrease of average noise PSD.

Since the noise effect on MIMO BPL capacity has been identified, only the average noise type will be adopted hereafter without affecting the generality of BPL capacity analysis concerning the influence of BPL noise models of the next subsection.

\section{The Role of Noise Models during Capacity Computations of OV HV MIMO BPL Networks}

Until now, it has been explicitly highlighted the tremendous broadband potential of OV HV power grid when MIMO technology is adopted. Despite these relatively favorable capacity characteristics, the influence of noise still remains a critical issue because its more disturbed versions may torpedo the whole broadband venture. Hence, due to its multi-Mbps impact, the use of accurate and simple noise models determines the accuracy of capacity computations [1].

To investigate the effect of different noise models on OV HV MIMO BPL capacity performance as well as the potential of using simple versions, such as the FL one, without, however, affecting the general capacity accuracy, a number of numerical results concerning capacities of OV HV MIMO BPL networks in the 3-88MHz frequency range are presented in this subsection. Note that during the following MIMO BPL capacity computations, only four representative MIMO transmission schemes are examined -i.e., SISO, 2x2 MIMO, 4x4 MIMO and 8x8 MIMO ones- in the name of terseness and simplicity, giving a representative image of the effect of noise models. 
In Figs. 5(a)-(f), the cumulative capacity is plotted versus frequency for the indicative OV HV BPL topologies, presented in Sec. IIB, when SISO transmission scheme is employed and the aforementioned seven well-known noise models are applied, respectively. In the same figures, the cumulative capacity percentage change (CCPC) between the respective noise models and FL one is presented. In Figs. 6(a)-(f), 7(a)-(f) and 8(a)-(f), same plots are given in the case of 2x2 MIMO, 4x4 MIMO and 8x8 MIMO transmission schemes, respectively.
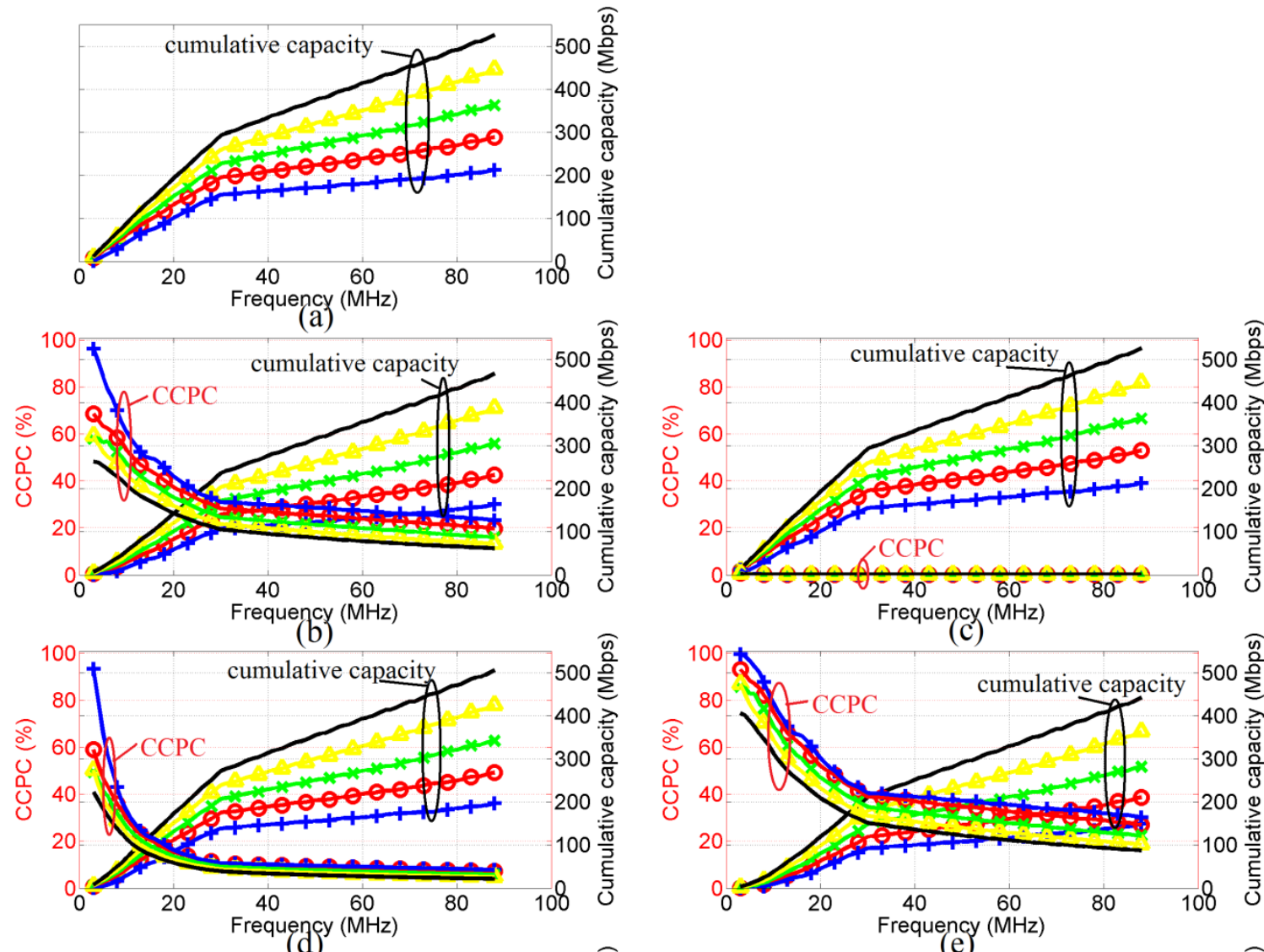

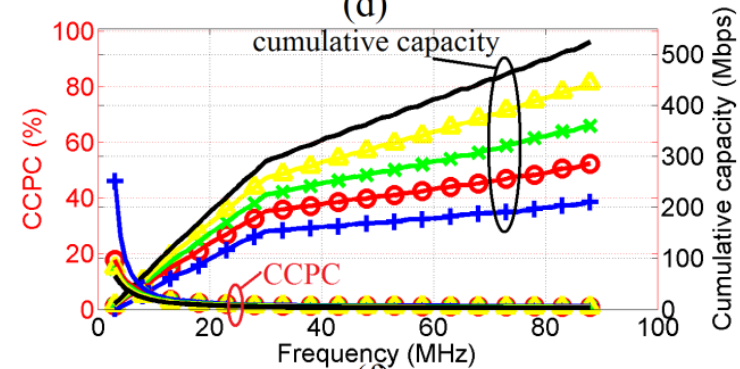

(f)

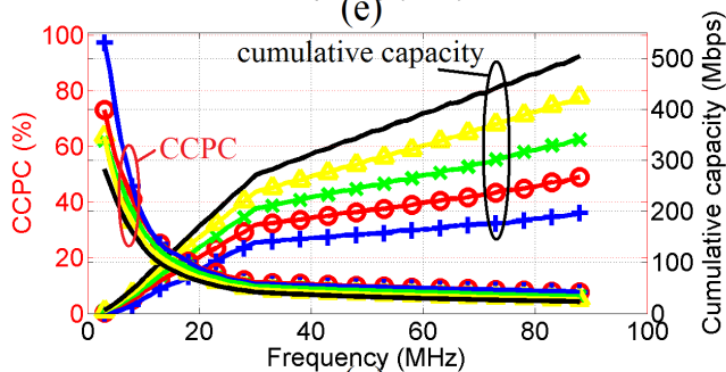

(g)

Figure 5. Cumulative capacity and CCPC versus frequency of the four indicative topologies of OV HV BPL networks -urban case A $(\theta)$, urban case B $(+)$, suburban case $(*)$, rural case $(\triangle)$, and "LOS" case $(-)$ - in the 3-88MHz frequency band for the seven aforementioned noise models when SISO transmission scheme is deployed, average noise type is assumed, and FCC Part 15 is adopted. (a) FL noise model (with no CCPC curves). (b) OPERA noise model. (c) MEN noise model. (d) PH1 noise model. (e) PH2 noise model. (f) ESM1 noise model. (g) ESM2 noise model. 

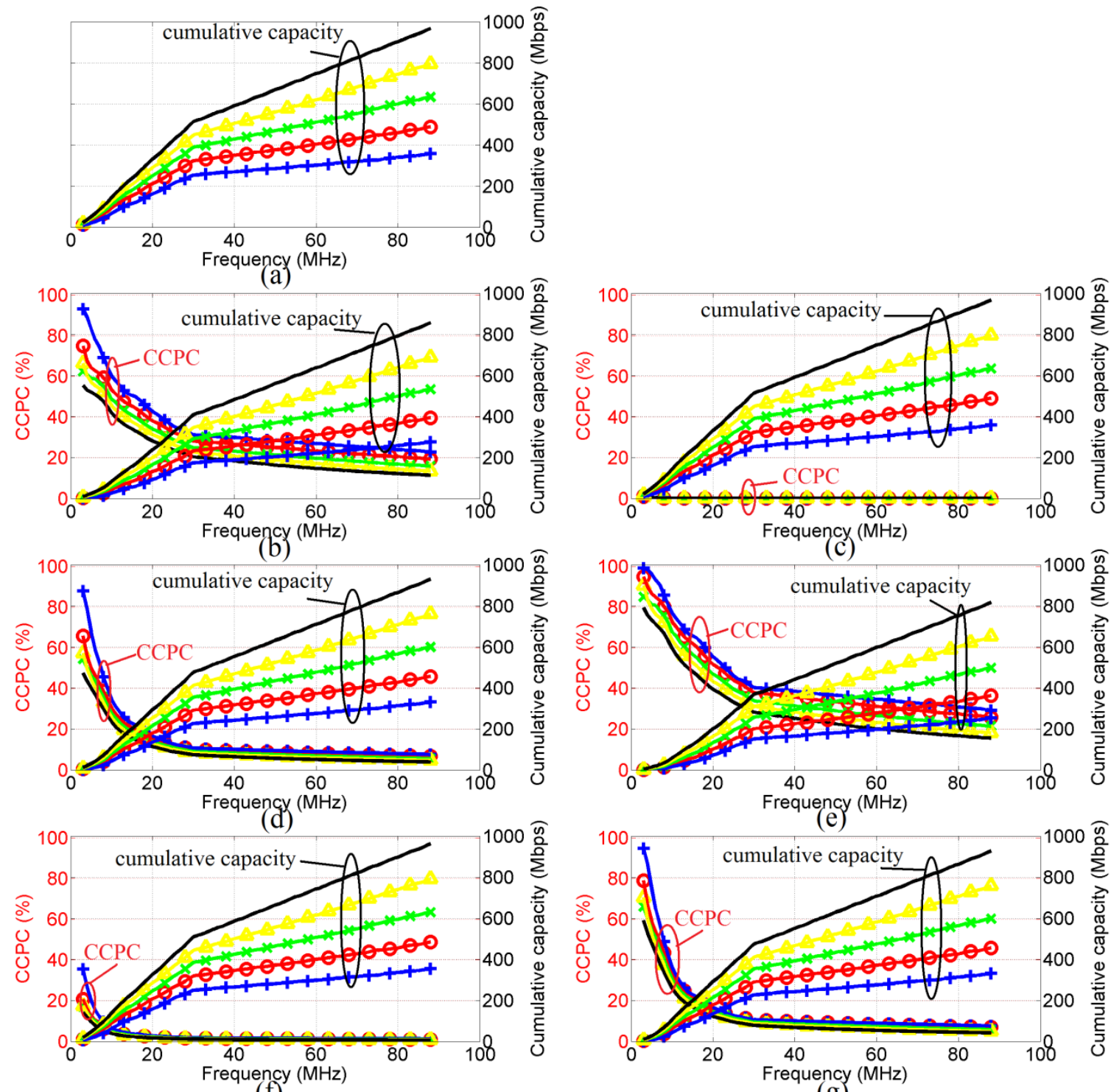

(f)

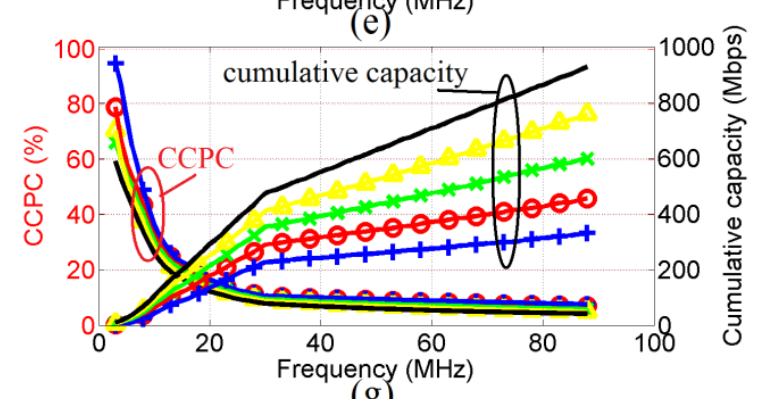

(g)

Figure 6. Same as in Fig. 5, but for 2x2 MIMO transmission scheme. 

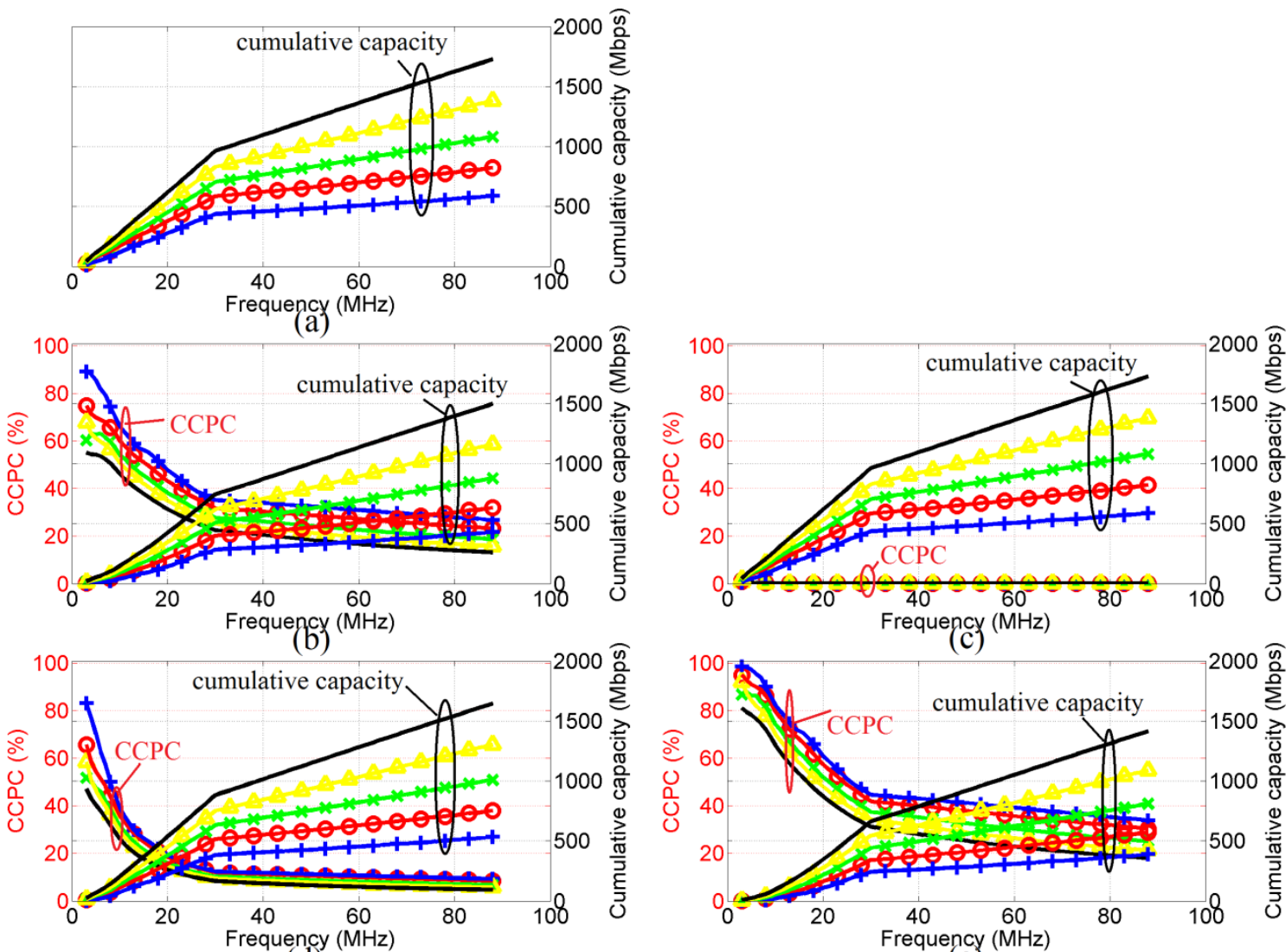

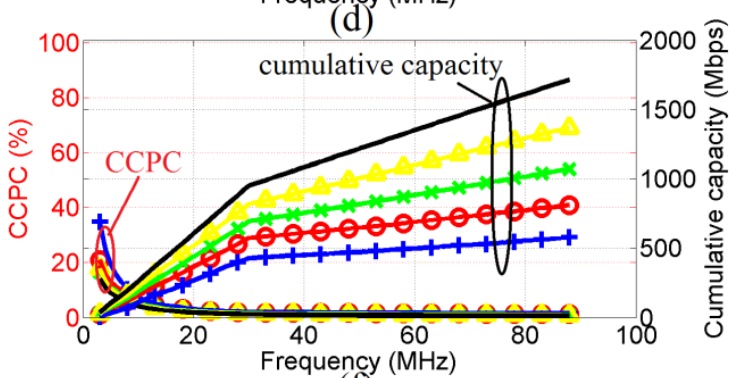

(f)

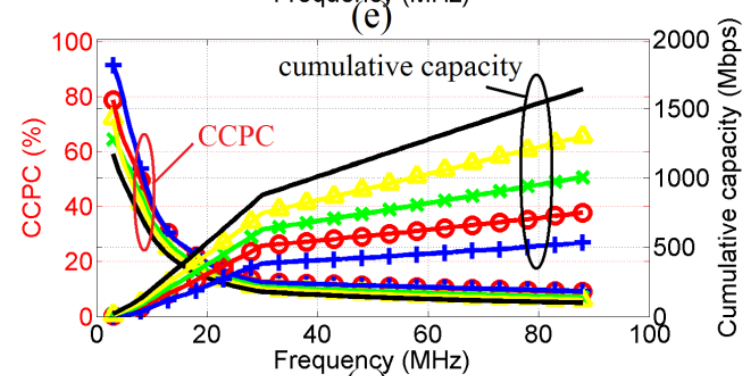

(g)

Figure 7. Same as in Fig. 5, but for $4 \times 4$ MIMO transmission scheme. 


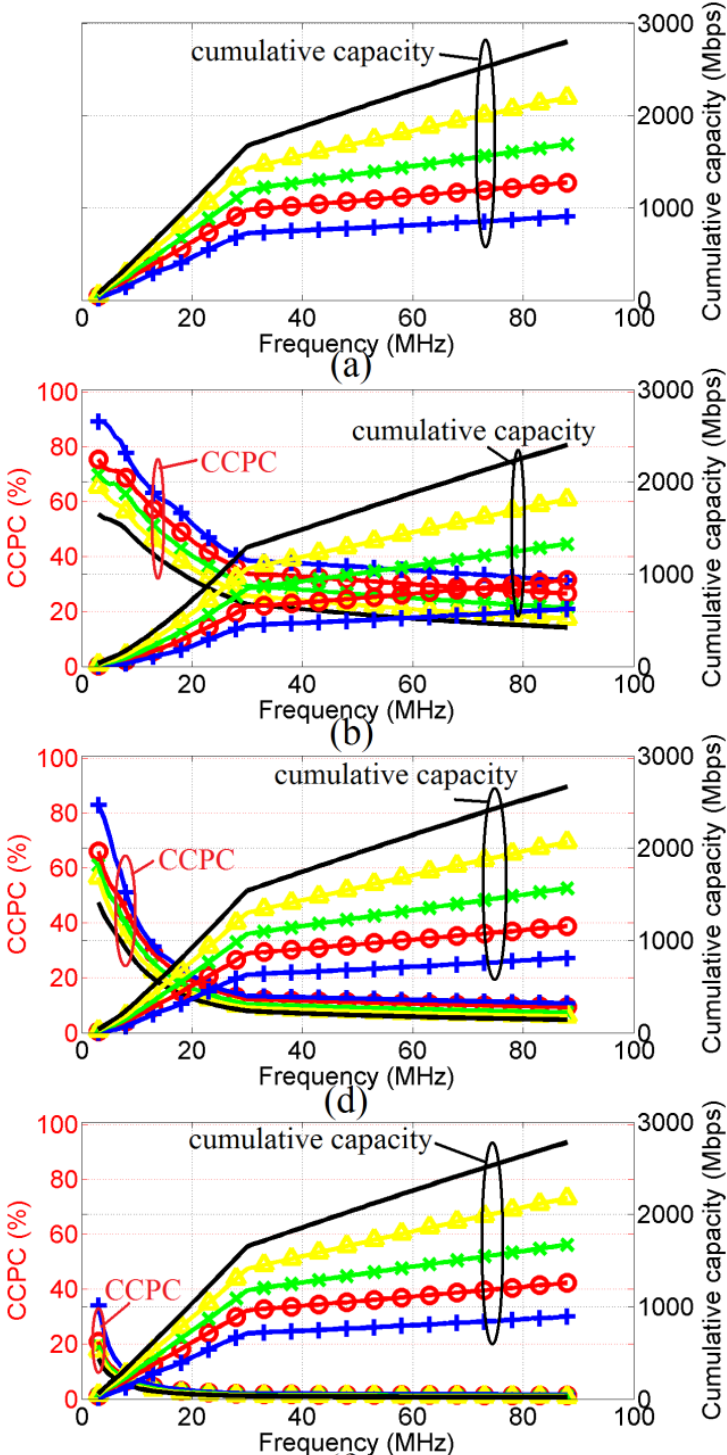

(f)

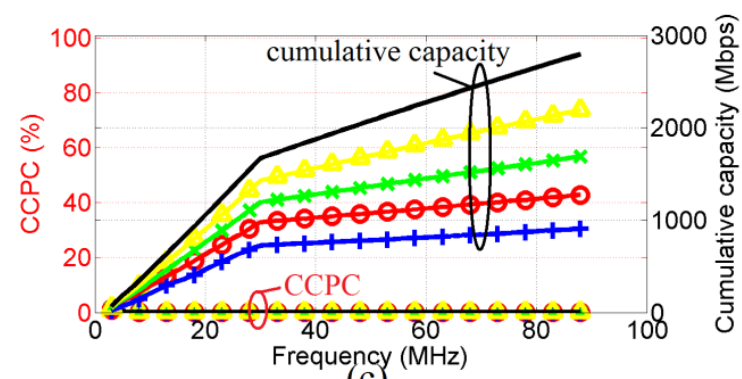

(c)

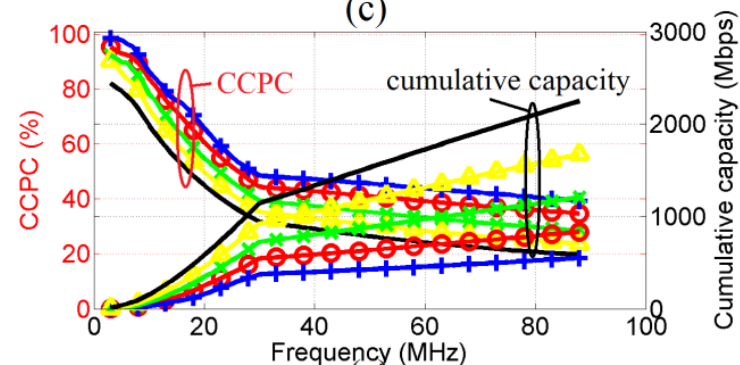

(e)

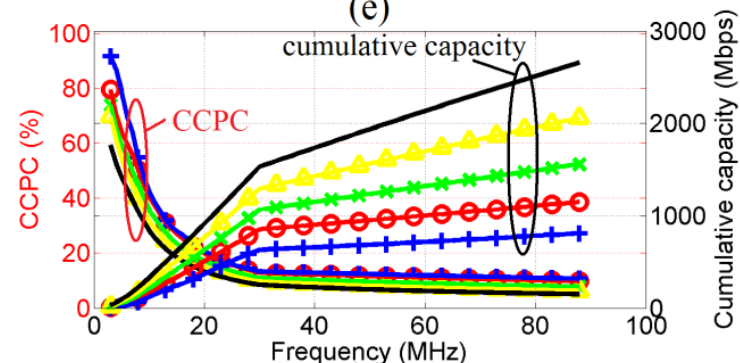

(g)

Figure 8. Same as in Fig. 3, but for 8x8 MIMO transmission scheme.

From Figs. 5(a)-(g), 6(a)-(g), 7(a)-(g) and 8(a)-(g) and taking under consideration the relevant results of [1], the following deductions are pointed out.

- CCPC curves validate the convergence of all well-known noise models. Actually, for frequencies less than a frequency threshold that is equal to approximately $30 \mathrm{MHz}$ for OV HV MIMO BPL networks, these networks suffer from correlated noise. This nonwhite noise profile generates the significant capacity differences in this frequency band. For frequencies above this frequency threshold, noise tends to follow the more commonly FL noise model while the capacity differences among different noise models become marginal. This noise behavior has also been validated among different OV and UN MV and LV SISO BPL networks in [1]. 
- CCPC curves also indicate that the aggravated topologies are more affected by either the noise fluctuations or the discrepancies of different noise models. Contrary to this behavior, "LOS" cases are characterized by higher immunity against the general issues of noise. In addition, this CCPC behavior among different topologies is independent of the applied MIMO transmission scheme.

- Apart from being inversely proportional to frequency, another interesting feature of CCPC curves is their relation with the full MIMO degree. Actually, there is a slight CCPC decrease with the increase of the degrees of MIMO transmission schemes at the lower frequencies whereas the opposite occurs in the higher part of BPL operation spectrum. This behavior has to do with the increased presence of all the modes supported by the MTL configuration and their diverse spectral behavior in the $3-88 \mathrm{MHz}$ frequency band [2]-[11], [28]. Anyway, due to its marginal change, CCPC may be considered as a constant percentage of capacity for given noise model and BPL topology, regardless of the applied MIMO transmission scheme. From Fig. 5(a)-(g), 6(a)-(g), 7(a)-(g) and 8(a)-(g), the average CCPC due to different noise models is approximately equal to $11 \%$; say, approximately $32 \mathrm{Mbps}$ average capacity loss per full MIMO degree.

- Comparing Figs. 5(a)-(g), 6(a)-(g), 7(a)-(g) and 8(a)-(g) with Figs. 4(a)-(h), the overall capacity aggravation of noise environments is higher compared with the overall capacity differences observed among the seven aforementioned noise models; as it has already been mentioned, each $\mathrm{dB}$ of noise PSD increase corresponds to an approximate $13 \mathrm{Mbps}$ capacity loss per full MIMO degree that is lower to the overall average capacity difference due to different noise models, which is equal to $32 \mathrm{Mbps}$. However, the deviation of PSD noise typically ranges above $\pm 3 \mathrm{~dB}$ with reference to the average noise PSD [2]-[13], [22], [23], [29], [30], [32]-[36], [44], [56]. Consequently, already verified in [1] for SISO BPL networks and subliminally reproduced among BPL engineers for years [4], [6], [12], [23], [29], [44], FL noise model may be comfortably used during capacity computations of all OV transmission MIMO BPL networks in the $3-88 \mathrm{MHz}$ frequency band. In the majority of the cases examined, the capacity differences among all available noise models remain satisfactorily low in this frequency band of BPL operation. The differences of FL noise model compared with specific noise models - e.g., OPERA, PH1, PH2 and ESM2 noise models- are concentrated on urban and industrial noise environments where OV transmission grids randomly occur, anyway. Therefore, similarly to SISO case in OV and UN distribution BPL networks, FL noise model may be safely used during capacity computations of all OV HV MIMO BPL networks in the $3-88 \mathrm{MHz}$ frequency range except for urban centers and heavy industrial zones where their disturbed noise conditions generate non negligible capacity differences among different noise models.

- Due to the common nature of noise, accurate and simple noise models may play the role of bridge among different SISO and MIMO distribution and transmission BPL networks offering an elementary step towards more coexistence among BPL systems in the oncoming smart grid network [61]-[63]. 


\section{Conclusions}

On the basis of [1], this paper has focused on the broadband potential of OV transmission MIMO BPL networks associated with the presence of different noise environments and noise models.

Through the prism of information theory, it has been verified that the capacity performance of OV transmission BPL networks is critically affected either by MIMO technology or by their surrounding noise conditions. Actually, the capacity impact of noise is so drastic that a slight increase of noise PSD in the order of few dBs may comminute the tremendous capacity boost that is generated by the deployment of MIMO systems; say, capacity fluctuations in the order of Gbps. Due to the high importance of noise, the development of simple and accurate noise models is imperative. Towards that direction, FL noise model, which has been proven to be an efficient and precise tool for computing the capacities of OV and UN distribution SISO BPL networks in the $3-88 \mathrm{MHz}$ frequency range (see in [1]), extends its applicability to OV HV MIMO BPL networks in the same frequency band that is the mainstream BPL operation spectrum. Actually, despite the slight capacity differences near urban and industrial zones, FL noise model exploits the converging behavior of all the well-validated BPL noise models in the $3-88 \mathrm{MHz}$ frequency band, giving decent capacity results, and simplifying, thus, the various capacity computations.

\section{Conflicts of Interest}

The author declares that there is no conflict of interests regarding the publication of this paper.

\section{References}

[1] A. G. Lazaropoulos, "The Impact of Noise Models on Capacity Performance of Distribution Broadband over Power Lines (BPL) Networks," Hindawi Computer Networks and Communications, vol. 2016, Article ID 5680850, 14 pages, 2016. DOI: $\quad 10.1155 / 2016 / 5680850 . \quad$ [Online]. Available: http://www.hindawi.com/journals/jenc/2016/5680850/

[2] A. G. Lazaropoulos, "Factors Influencing Broadband Transmission Characteristics of Underground Low-Voltage Distribution Networks," IET Commun., vol. 6, no. 17, pp. 2886-2893, Nov. 2012. DOI: 10.1049/ietcom.2011.0661

[3] A. G. Lazaropoulos and P. G. Cottis, "Transmission characteristics of overhead medium voltage power line communication channels," IEEE Trans. Power Del., vol. 24, no. 3, pp. 1164-1173, Jul. 2009. DOI: 10.1109/TPWRD.2008.2008467

[4] A. G. Lazaropoulos and P. G. Cottis, "Capacity of overhead medium voltage power line communication channels," IEEE Trans. Power Del., vol. 25, no. 2, pp. 723-733, Apr. 2010. DOI: 10.1109/TPWRD.2009.2034907

[5] A. G. Lazaropoulos and P. G. Cottis, "Broadband transmission via underground medium-voltage power lines-Part I: transmission characteristics," IEEE Trans. Power Del., vol. 25, no. 4, pp. 2414-2424, Oct. 2010. DOI: 10.1109/TPWRD.2010.2048929 
[6] A. G. Lazaropoulos and P. G. Cottis, "Broadband transmission via underground medium-voltage power lines-Part II: capacity," IEEE Trans. Power Del., vol. 25, no. 4, pp. 2425-2434, Oct. 2010. DOI: 10.1109/TPWRD.2010.2052113

[7] A. G. Lazaropoulos, "Broadband transmission characteristics of overhead highvoltage power line communication channels," Progress in Electromagnetics Research B, vol. 36, pp. 373-398, 2012. DOI: 10.2528/PIERB11091408 [Online]. Available: http://www.jpier.org/PIERB/pierb36/19.11091408.pdf

[8] A. G. Lazaropoulos, "Towards broadband over power lines systems integration: Transmission characteristics of underground low-voltage distribution power lines," Progress in Electromagnetics Research B, 39, pp. 89-114, 2012. DOI: 10.2528/PIERB12012409 [Online].

Available: http://www.jpier.org/PIERB/pierb39/05.12012409.pdf

[9] A. G. Lazaropoulos, "Broadband transmission and statistical performance properties of overhead high-voltage transmission networks," Hindawi Journal of Computer Networks and Commun., 2012, article ID 875632, 2012. DOI: 10.1155/2012/875632 [Online]. Available: http://www.hindawi.com/journals/jcnc/aip/875632/

[10] A. G. Lazaropoulos, "Towards modal integration of overhead and underground low-voltage and medium-voltage power line communication channels in the smart grid landscape: model expansion, broadband signal transmission characteristics, and statistical performance metrics (Invited Paper)," ISRN Signal Processing, vol. 2012, Article ID 121628, pp. 1-17, 2012. DOI: 10.5402/2012/121628 [Online]. Available: http://www.hindawi.com/journals/isrn/2012/121628/

[11] A. G. Lazaropoulos, "Review and Progress towards the Common Broadband Management of High-Voltage Transmission Grids: Model Expansion and Comparative Modal Analysis," ISRN Electronics, vol. 2012, Article ID 935286, pp. 1-18, 2012. DOI: 10.5402/2012/935286 [Online]. Available: http://www.hindawi.com/isrn/electronics/2012/935286/

[12] A. G. Lazaropoulos, "Review and Progress towards the Capacity Boost of Overhead and Underground Medium-Voltage and Low-Voltage Broadband over Power Lines Networks: Cooperative Communications through Two- and ThreeHop Repeater Systems," ISRN Electronics, vol. 2013, Article ID 472190, pp. 1-19, 2013. DOI: 10.1155/2013/472190 [Online]. Available: http://www.hindawi.com/journals/isrn/2013/472190/

[13] A. G. Lazaropoulos, "Green Overhead and Underground Multiple-Input MultipleOutput Medium Voltage Broadband over Power Lines Networks: EnergyEfficient Power Control," Springer Journal of Global Optimization, vol. 2012 / Print ISSN 0925-5001, pp. 1-28, Oct. 2012. DOI: 10.1007/s10898-012-9988-y

[14] A. Milioudis, G. T. Andreou, and D. P. Labridis, "Detection and location of high impedance faults in multiconductor overhead distribution lines using power line communication devices," IEEE Trans. on Smart Grid, vol. 6, no. 2, pp. 894-902, 2015. DOI: 10.1109/TSG.2014.2365855

[15] G. C. Christoforidis, I. P. Panapakidis, T. A. Papadopoulos, G. K. Papagiannis, I. Koumparou, M. Hadjipanayi, and G. E. Georghiou, "A Model for the Assessment of Different Net-Metering Policies," Energies, vol. 9, no. 4, pp. 1-24, 2016. DOI: $10.3390 /$ en 9040262 
[16] M. Biagi, "MIMO self-interference mitigation effects on power line relay networks," IEEE Commun. Lett., vol. 15, no. 8, pp. 866-868, Aug. 2011. DOI: 10.1109/LCOMM.2011.062911.110230

[17] D. Schneider, A. Schwager, J. Speidel and A. Dilly, "Implementation and results of a MIMO PLC feasibility study," in Proc. IEEE Int. Symp. Power Line Communications and Its Applications, Udine, Italy, Apr. 2011, pp. 54-59. DOI: 10.1109/ISPLC.2011.5764450

[18] A. Schwager, D. Schneider, W. Bäschlin, A. Dilly, and J. Speidel, "MIMO PLC: Theory, Measurements and System Setup," in Proc. IEEE Int. Symp. Power Line Communications and Its Applications, Udine, Italy, Apr. 2011, pp. 48-53. DOI: 10.1109/ISPLC.2011.5764447

[19] F. Versolatto and A. M. Tonello, "An MTL theory approach for the simulation of MIMO power-line communication channels," IEEE Trans. Power Del., vol. 26, no. 3, pp. 1710-1717, Jul. 2011. DOI: 10.1109/TPWRD.2011.2126608

[20] A. Canova, N. Benvenuto, and P. Bisaglia, "Receivers for MIMO-PLC channels: Throughput comparison," in Proc. IEEE Int. Symp. Power Line Communications and Its Applications, Rio de Janeiro, Brazil, Mar. 2010, pp. 114-119. DOI: 10.1109/ISPLC.2010.5479904

[21] D. Schneider, J. Speidel, L. Stadelmeier, and D. Schill, "Precoded spatial multiplexing MIMO for inhome power line communications," in Proc. IEEE Global Telecommunications Conference, New Orleans, LA, USA, Nov./Dec. 2008, pp. 1-5. DOI: 10.1109/GLOCOM.2008.ECP.556

[22] M. Zimmermann and K. Dostert, "Analysis and modeling of impulsive noise in broad-band powerline communications," IEEE Trans. Electromagn. Compat., vol. 44, no. 1, pp. 249-258, Feb. 2002. DOI: 10.1109/15.990732

[23] P. Amirshahi and M. Kavehrad, "High-frequency characteristics of overhead multiconductor power lines for broadband communications," IEEE J. Sel. Areas Commun., vol. 24, no. 7, pp. 1292-1303, Jul. 2006. DOI: 10.1109/JSAC.2006.874399

[24] T. Sartenaer, "Multiuser communications over frequency selective wired channels and applications to the powerline access network" Ph.D. dissertation, Univ. Catholique Louvain, Louvain-la-Neuve, Belgium, Sep. 2004.

[25] T. Calliacoudas and F. Issa, "“'Multiconductor transmission lines and cables solver," An efficient simulation tool for plc channel networks development," presented at the IEEE Int. Conf. Power Line Communications and Its Applications, Athens, Greece, Mar. 2002.

[26] A. G. Lazaropoulos, "Policies for Carbon Energy Footprint Reduction of Overhead Multiple-Input Multiple-Output High Voltage Broadband over Power Lines Networks," Trends in Renewable Energy, vol. 1, no. 2, pp. 87-118, Jun. 2015. DOI: 10.17737/tre.2015.1.2.0011 [Online]. Available: http://futureenergysp.com/index.php/tre/article/view/11/17

[27] T. Sartenaer and P. Delogne, "Deterministic modelling of the (Shielded) outdoor powerline channel based on the multiconductor transmission line equations," IEEE J. Sel. Areas Commun., vol. 24, no. 7, pp. 1277-1291, Jul. 2006. DOI: 10.1109/JSAC.2006.874423

[28] C. R. Paul, Analysis of Multiconductor Transmission Lines. New York: Wiley, 1994. 
[29] Y. H. Kim, S. Choi, S. C. Kim, and J. H. Lee, "Capacity of OFDM two-hop relaying systems for medium-voltage power-line access networks," IEEE Trans. Power Del., vol. 27, no. 2, pp. 886-894, Apr. 2012. DOI: 10.1109/TPWRD.2011.2182063

[30] OPERA1, D5: Pathloss as a function of frequency, distance and network topology for various LV and MV European powerline networks. IST Integrated Project No 507667, Apr. 2005.

[31] K. Dostert, Powerline Communications. Upper Saddle River, NJ: Prentice-Hall, 2001 .

[32] Ofcom, "DS2 PLT Measurements in Crieff," Ofcom, Tech. Rep. 793 (Part 2), May 2005, [Online]. Available: http://www.ofcom.org.uk/research/technology/research/archive/cet/powerline/ds2. pdf

[33] Ofcom, “Ascom PLT Measurements in Winchester," Ofcom, Tech. Rep. 793 (Part 1), May 2005, [Online]. Available: http://www.ofcom.org.uk/research/technology/research/archive/cet/powerline/asc om.pdf

[34] M. Katayama, T. Yamazato, and H. Okada, "A mathematical model of noise in narrowband power line communication systems," IEEE J. Sel. Areas Commun., vol. 24, no. 7, pp. 1267-1276, Jul. 2006. DOI: 10.1109/JSAC.2006.874408

[35] J. Song, C. Pan, Q. Wu, Z. Yang, H. Liu, B. Zhao, and X. Li, "Field Trial of Digital Video Transmission over Medium-Voltage Powerline with Time-Domain Synchronous Orthogonal Frequency Division Multiplexing Technology," in Proc. 2007 IEEE Int. Symp. on Power Line Communications and its Applications, Pisa, Italy, Mar. 2007, pp. 559-564.

[36] H. Liu, J. Song, B. Zhao, and X. Li, "Channel study for medium-voltage power network," in Proc. 2006 IEEE International Symposium on Power Line Communications and its Applications, Orlando, FL, USA, Mar. 2006, pp. 245-250. DOI: 10.1109/ISPLC.2006.247469

[37] V. Degardin, M. Lienard, A. Zeddam, F. Gauthier, and P. Degauque, "Classification and characterization of impulsive noise on indoor power line used for data communications," IEEE Trans. on Consumer Electronics, vol. 48, no. 4, pp. 913-918, 2002. DOI: 10.1109/TCE.2003.1196420

[38] F. Cañete, J. Cortés, L. Díez, and J. Entrambasaguas, "Analysis of the cyclic short-term variation of indoor power line channels," IEEE J. Sel. Areas Commun., vol. 24, no. 7, pp. 1327-1338, Jul. 2006. DOI: 10.1109/JSAC.2006.874402

[39] H. Meng, Y. L. Guan, and S. Chen, "Modeling and analysis of noise effects on broadband power line communications," IEEE Trans. Power Del., vol. 20, no. 2, pp. 630-637, Apr. 2005. DOI: 10.1109/TPWRD.2005.844349

[40] M. V. Ribeiro, C. A. Duque, and J. M. T. Romano, "An interconnected type-1 fuzzy algorithm for impulsive noise cancellation in multicarrier-based power line communication systems," IEEE Journal on Sel. Areas in Comm., vol. 24, no. 7, pp. 1364-1376, July 2006. DOI: 10.1109/JSAC.2006.874417

[41] H. Hrasnica, A. Haidine, and R. Lehnert, Broadband Powerline Communications: Network Design. Wiley, 2004.

[42] J. Meng and A. E. Marble, "Effective communication strategies for noise-limited power-line channels," IEEE Trans. on Power Del., vol. 22, no. 2, pp. 887-892, April 2007. DOI: 10.1109/TPWRD.2006.883018 
[43] J. Zhang and J. Meng, "Noise Resistant OFDM for Power-Line Communication Systems," IEEE Trans. on Power Del., vol. 25, no. 2, pp. 693-710, Apr. 2010. DOI: 10.1109/TPWRD.2009.2036626

[44] P. Amirshahi, "Broadband access and home networking through powerline networks" Ph.D. dissertation, Pennsylvania State Univ., University Park, PA, May 2006.

Available:

http://etda.libraries.psu.edu/theses/approved/WorldWideIndex/ETD1205/index.html

[45] N. Suljanović, A. Mujčić, M. Zajc, and J. F. Tasič, “Approximate computation of high-frequency characteristics for power line with horizontal disposition and middle-phase to ground coupling," Elsevier Electr. Power Syst. Res., vol. 69, pp. 17-24, Jan. 2004. DOI: 10.1016/j.epsr.2003.07.005

[46] M. D'Amore and M. S. Sarto, "Simulation models of a dissipative transmission line above a lossy ground for a wide-frequency range-Part I: Single conductor configuration," IEEE Trans. Electromagn. Compat., vol. 38, no. 2, pp. 127-138, May 1996. DOI: $10.1109 / 15.494615$

[47] M. D'Amore and M. S. Sarto, "Simulation models of a dissipative transmission line above a lossy ground for a wide-frequency range-Part II: Multi-conductor configuration," IEEE Trans. Electromagn. Compat., vol. 38, no. 2, pp. 139-149, May 1996. DOI: $10.1109 / 15.494616$

[48] OPERA1, D44: Report presenting the architecture of plc system, the electricity network topologies, the operating modes and the equipment over which PLC access system will be installed, IST Integr. Project No 507667, Dec. 2005.

[49] R. Pighi and R. Raheli, "On Multicarrier Signal Transmission for High-Voltage Power Lines," in Proc. IEEE Int. Symp. Power Line Commun. Appl., Vancouver, BC, Canada, Apr. 2005, pp. 32-36.

[50] N. Theethayi, "Electromagnetic interference in distributed outdoor electrical systems, with an emphasis on lightning interaction with electrified railway network," Ph.D. dissertation, Uppsala Univ., Uppsala, Sweden, Sep. 2005, [Online]. Available: http://uu.divaportal.org/smash/get/diva2:166746/FULLTEXT01

[51] D. Schneider, A. Schwager, W. Baschlin, and P. Pagani, "European MIMO PLC field measurements: Channel analysis," in Proc. IEEE Int. Symp. Power Line Commun. Appl., Beijing, China, Mar. 2012, pp. 304-309. DOI: 10.1109/ISPLC.2012.6201316

[52] NATO, "HF Interference, Procedures and Tools (Interférences HF, procédures et outils) Final Report of NATO RTO Information Systems Technology," RTO-TRISTR-050, Jun. 2007, [Online]. Available: http://ftp.rta.nato.int/public/PubFullText/RTO/TR/RTO-TR-IST-050/\$\$TR-IST050-ALL.pdf

[53] FCC, "In the Matter of Amendment of Part 15 regarding new requirements and measurement guidelines for Access Broadband over Power Line Systems," FCC 04-245 Report and Order, Jul. 2008.

[54] A. Milioudis, G. Andreou, and D. Labridis, "Optimum transmitted power spectral distribution for broadband power line communication systems considering electromagnetic emissions," Elsevier Electric Power Systems Research, 2016. DOI: $10.1016 /$ j.epsr.2016.03.047 
[55] O. G. Hooijen, "On the channel capacity of the residential power circuit used as a digital communications medium," IEEE Commun. Lett., vol. 2, no. 10, pp. 267268, Oct. 1998. DOI: 10.1109/4234.725218

[56] D. Benyoucef, "A new statistical model of the noise power density spectrum for powerline communications," in IEEE Proceedings of the International Symposium on Power-Line Communications and its Applications, Kyoto, Japan, Mar. 2003, pp. 136-141.

[57] H. Philipps, "Development of a statistical model for powerline communications channels," presented in IEEE Proceedings of the International Symposium on Power-Line Communications and its Applications, Limerick, Ireland, Apr. 2000.

[58] T. Esmailian, F. R. Kschischang, and P. G. Gulak, "In-building power lines as high-speed communication channels: Channel characterization and a test channel ensemble," Int. J. Commun. Syst., vol. 16, pp. 381-400, May 2003. DOI: 10.1002/dac.596

[59] A. G. Lazaropoulos, "Wireless Sensor Network Design for Transmission Line Monitoring, Metering and Controlling: Introducing Broadband over PowerLinesenhanced Network Model (BPLeNM)," ISRN Power Engineering, vol. 2014, Article ID 894628, 22 pages, 2014. DOI: 10.1155/2014/894628. [Online]. Available:

http://www.hindawi.com/journals/isrn.power.engineering/2014/894628/

[60] A. G. Lazaropoulos, "Wireless Sensors and Broadband over PowerLines Networks: The Performance of Broadband over PowerLines-enhanced Network Model (BPLeNM) (Invited Paper)," ICAS Publishing Group Transaction on IoT and Cloud Computing, vol. 2, no. 3, pp. 1-35, 2014. [Online]. Available: http://icas-pub.org/ojs/index.php/ticc/article/view/27/17

[61] A. G. Lazaropoulos, "Designing Broadband over Power Lines Networks Using the Techno-Economic Pedagogical (TEP) Method - Part II: Overhead LowVoltage and Medium-Voltage Channels and Their Modal Transmission Characteristics," Trends in Renewable Energy, vol. 1, no. 2, pp. 59-86, Jun. 2015. DOI: $\quad 10.17737 /$ tre.2015.1.2.006 [Online]. Available: http://futureenergysp.com/index.php/tre/article/view/6/16

[62] E. A. Bakirtzis, C. K. Simoglou, P. N. Biskas, D. P. Labridis, and A. G. Bakirtzis, "Comparison of advanced power system operations models for large-scale renewable integration," Elsevier Electric Power Systems Research, vol. 128, pp. 90-99, 2015. DOI: 10.1016/j.epsr.2015.06.025

[63] A. G. Lazaropoulos, "Designing Broadband over Power Lines Networks Using the Techno-Economic Pedagogical (TEP) Method - Part I: Overhead High Voltage Networks and Their Capacity Characteristics," Trends in Renewable Energy, vol. 1, no. 1, pp. 16-42, Mar. 2015. DOI: 10.17737/tre.2015.1.1.002 [Online]. Available: http://futureenergysp.com/index.php/tre/article/view/2

Article copyright: (C) 2016 Athanasios G. Lazaropoulos. This is an open access article distributed under the terms of the Creative Commons Attribution 4.0 International License, which permits unrestricted use and distribution provided the original author and source are credited. 